\title{
Comment on "Short peptide analogs as alternatives to collagen in pro-regenerative corneal implants" by Jangamreddy JR et al.
}

Jaywant Phopase

The self-archived postprint version of this journal article is available at Linköping University Institutional Repository (DiVA):

http://urn.kb.se/resolve?urn=urn:nbn:se:liu:diva-162349

N.B.: When citing this work, cite the original publication.

Phopase, J., (2019), Comment on "Short peptide analogs as alternatives to collagen in pro-regenerative corneal implants" by Jangamreddy JR et al., Acta Biomaterialia, 97, 691-691.

https://doi.org/10.1016/j.actbio.2019.06.004

Original publication available at:

https://doi.org/10.1016/j.actbio.2019.06.004

Copyright: Elsevier

http://www.elsevier.com/

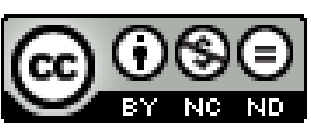




\section{Dear Editor-in-Chief, Acta Biomaterialia,}

I am writing to you in regarding an article entitled " Short peptide analogs as alternatives to collagen in pro-regenerative corneal implants" by Jangamreddy JR et.al, published in your journal. The research work published in this article was performed in collaboration between me and Dr. Griffith at Linköping University. The original concept, design, fabrication and characterization of the peptides and the materials implanted into the mini pigs, rabbits and in vitro studies originated from my research. This research was funded by Swedish research council (VR) through a junior researcher grant to me and the animal studies were funded by joint Vinnova Indo-Sweden grant to me, Dr. Fagerholm and Dr. Griffith. Some of the materials included in this article have been patented (GB2540116B), where I am the sole inventor. Furthermore, some of the results presented in this article were presented at the Association for Research in Vision and Ophthalmology 2016 annual meeting, where my student Ranjithkumar Ravichandran and I were listed in the author list. Considering the guidelines provided by the International Committee of Medical Journal Editors, my student and I have certainly made a significant contribution to justify authorship.

I would also like to bring the following major scientific concerns to your notice regarding this manuscript.

1) The materials implanted into mini pigs, rabbits and materials used for the reported in vitro studies differ from each other. In the material and methods section, the authors state that the materials were produced by company, UAB Ferentis, Vilnius, Lithuania. These materials were designed and synthesized by me at Linköping University. This company did not exist when we implanted the materials into the pigs.

2) The TEM image (Figure 1d) provided in the article does not appear to show signs of a fibril structure. In my own experience using other experimental methods (cryo SEM, DLS) fibril structure was never observed with the collagen-like peptide conjugated to poly(ethylene glycol) (CLP-PEG) material.

3) Figure 1e, in the article shows that the storage modulus of the CLP hydrogel is higher than native rabbit cornea. This indicates that CLP-PEG hydrogels are stronger than rabbit cornea which is contradictory to the tensile strength results presented in the same manuscript. The tensile strength of CLP-PEG hydrogel is only $0.07 \mathrm{MPa}$ (Please see the supporting info of the article: Table S2: Properties of CLP-PEG hydrogels). The tensile strength for native rabbit cornea is $3.8-5 \mathrm{MPa}$ and therefore, the very low storage modulus $(\approx 1000 \mathrm{~Pa})$ reported in the article for native rabbit cornea does not make sense. These results are also in contradiction to our previous report (RSC Adv., 2016, 6, 55745, Table 1), were we reported the Young's modulus of CLP-PEG hydrogel in comparison to human cornea. We observed that the modulus of human cornea is several folds higher (3-13 Mpa) compared to CLP-PEG hydrogel $(0.18 \mathrm{MPa})$, indicating higher stiffness of human cornea. However, in the current article, authors have concluded exactly the opposite (CLPPEG hydrogels showed that the storage modulus values of the implants were much higher than those of the rabbit corneas, indicating higher stiffness).

The CLP-PEG hydrogel and rabbit cornea are different materials with different elastic deformation and mechanical strength. These materials will have a different degree of deformation 
at the same applied force and therefore, it does not make sense to compare the viscoelastic properties of these materials using the same rheology parameters as used by the authors in this article. Please also note that the shape and thickness of the rabbit cornea and CLP-PEG samples used for rheological measurements were not the same and therefore will affect the rheological measurements. Therefore, the reported comparison of storage modulus of the CLP hydrogel and rabbit cornea is incorrect.

4) Previous reports on recombinant human collagen type III (RHC-III)-based corneal implants show that the implants were observed even four years after they were implanted into humans (Biomaterials, 2014, 35, 2420-2427). The major content of CLP-PEG is PEG, which is a synthetic polymer and therefore, it does not seem logical to conclude that CLP-PEG implants were remodeled and facilitated the regeneration of the native cornea in 12 months. In our experience (RSC Adv., 2016, 6, 55745), subcutaneous implantation of CLP-PEG hydrogels into the dorsum of rats for 90 days were intact without any sign of degradation. Furthermore, CLP-PEG hydrogel showed complete resistance to degradation when subjected to high concentrations of collagenase. We also observed the presence of PEG polymers, 12 months after grafting with CLP-PEG implants in mini pigs.

Sincerely,

Jaywant Phopase, PhD, Docent

Department of Physics, Chemistry and Biology,

Linköping University, Sweden

E-mail address: jaywant.phopase@liu.se 